Check for updates

Cite this: RSC Adv., 2019, 9, 18018

\title{
Lysine-based dendrimer with double arginine residues $\uparrow$
}

\author{
Nadezhda N. Sheveleva, (D) *a Denis A. Markelov, (D) a Mikhail A. Vovk, (D) a \\ Mariya E. Mikhailova, (D) a Irina I. Tarasenko, ${ }^{b}$ Peter M. Tolstoy, (DD ${ }^{a}$ Igor M. Neelov (D) ${ }^{c}$ \\ and Erkki Lähderanta ${ }^{d}$
}

Due to their well-defined structure, multivalency, biocompatibility, and low toxicity, lysine dendrimers can be used as safe and efficient nanocarriers for drug and gene delivery. One useful strategy for improving the gene delivery properties of dendrimers is modification with arginine amino acid (Arg) residues. Incorporation of Arg residues could be favorable for the enhancement in transfection efficiency of lysine based dendrimers. In this work, we have synthesized a new second-generation poly-L-lysine dendrimer with repeating units containing two linear Arg residues between neighboring lysine branching points (Lys-2Arg dendrimer) and studied its physicochemical properties. We confirmed the structure of Lys2Arg dendrimer using various one- and two-dimensional ${ }^{1} \mathrm{H}$ and ${ }^{13} \mathrm{C}$ NMR spectroscopy methods. Comparison of $T_{1 \mathrm{H}}$ relaxation data for Lys-2Arg and Lys-2Lys dendrimers showed that the replacement of double Lys residues with double Arg residues resulted in a sharp decrease in the mobility of methylene groups in side segments and in the main chain of $\varepsilon$-Lys inner segments. We suggest that this unexpected effect is caused by a guanidine-guanidine pairing effect in water, which leads to entanglements between dendrimer branches.

\author{
Received 1st April 2019 \\ Accepted 29th May 2019 \\ DOI: 10.1039/c9ra02461a \\ rsc.li/rsc-advances
}

\section{Introduction}

Dendrimers are nanosized artificial macromolecules with welldefined globular architecture that possess a variety of unique properties. ${ }^{1,2}$ They have a regular hyperbranched structure with a hydrophobic and/or hydrophilic interior, multivalency and extremely low polydispersity, which make them very suitable as nanocontainers for substances of different natures: from metal particles to genes and drugs. ${ }^{3-6}$ Every year, the number of papers dedicated to the study of the properties of dendrimers and their application in biomedicine and pharmaceutics increases., ${ }^{7,8}$ This trend demonstrates the importance of searching for dendrimers of new compositions and architectures to achieve safer and more efficient drug and gene delivery.,

Among a wide variety of dendrimers, poly-L-lysine (PLL) dendrimers have attracted especial attention from researchers

${ }^{a}$ St. Petersburg State University, 7/9 Universitetskaya Nab., St. Petersburg, 199034 Russia. E-mail: n.n.sheveleva@spbu.ru

${ }^{b}$ Institute of Macromolecular Compounds, Russian Academy of Sciences, Bolshoi Prospect 31, V.O., St. Petersburg, 199004 Russia. E-mail: itarasenko@list.ru

'St. Petersburg National Research University of Information Technologies, Mechanics and Optics (ITMO University), Kronverkskiy Pr. 49, St. Petersburg, 197101 Russia. E-mail:i.neelov@mail.ru

${ }^{d}$ Department of Physics, LUT University, Box 20, 53851 Lappeenranta, Finland. E-mail: erkki.lahderanta@lut.fi

$\dagger$ Electronic supplementary information (ESI) available. See DOI: 10.1039/c9ra02461a due to their high biocompatibility and relatively low toxicity. PLL dendrimers are modified with anionic, cationic and neutral amino acid residues to enhance their drug and gene delivery potential. The most common means of functionalization is through conjugation of other amino acid residues to the dendrimer terminal groups. ${ }^{11}$ The dendrimer interior can also be modified by incorporation of amino acid residues between inner lysine branching points. ${ }^{12}$

Functionalization of a PLL dendrimer with arginine (Arg) residues improves its transfection properties, thus increasing the charge of the dendrimer. ${ }^{11,12}$ Arginine residues are positively charged and protonated under biological conditions due to the presence of guanidine groups. ${ }^{13}$ Decoration of the dendrimer periphery with Arg residues improves its interaction with a negatively charged cell membrane, facilitating membrane penetration and enhancing transfection efficiency. ${ }^{5,9,14-18}$ It has been shown that guanidine groups have high binding ability to phosphate groups of DNA, which increases the condensation of DNA on the dendrimer surface..$^{5,9,11}$ The presence of arginine moieties in peptides improves cellular uptake of siRNA. ${ }^{13,19}$ Additionally, when we introduce charged double Arg residues between inner Lys branching points of PLL dendrimer, we make its interior more charged and thus more hydrophilic. Due to this reason, such modified arginine-rich dendrimer is less suitable for encapsulation of hydrophobic drugs but is of great interest for gene delivery. Transfection is enhanced by increasing the cationic charge of dendrimer. ${ }^{19}$ In the work, ${ }^{20}$ 
dendrimers with inner segments containing lysine-leucine or arginine-leucine amino acid residues were synthesized and investigated. It was found that dendrimers with Arg residues in the inner segments exhibited three-fold higher DNA transfection than dendrimers with Lys residues.

The aim of the present work is to take the first steps in studying a new poly-L-lysine dendrimer containing double Arg residues with guanidine groups in side segments (Fig. 1 and 2). We have attached arginine residues to the inner segments, rather than to the terminal groups, of the dendrimer, i.e. inserting arginine residues in each inner dendrimer shells except the terminal shell. This structure makes a dendrimer more cationic and can contribute to a more uniform distribution of charged groups in the volume of the dendrimer that should result in better transfection efficiency. ${ }^{20}$ At the same time, Arg residues in dendrimer interior should be available to water and other small molecules because it is well known that about $70 \%$ of the volume inside the lysine dendrimers is occupied by water. ${ }^{21}$ We believe that the introduction of arginine into the dendrimer interior will significantly improve the delivery of RNA and DNA and promote the formation of a more stable complex between dendrimers and RNA/DNA molecules and an increase in their transfection. In this paper, we perform the synthesis of arginine-modified poly-L-lysine dendrimer as well as confirm and characterize its structure and physicochemical properties by ${ }^{1} \mathrm{H}$ and ${ }^{13} \mathrm{C}$ NMR spectroscopy.

\section{Experimental}

Lysine-based second-generation dendrimer with inserted Arg residues (Lys-2Arg dendrimer) was synthesized by standard solid phase peptide synthesis (SPPS) (see ESI $\dagger$ ). The structure of the investigated dendrimer is shown in Fig. 2. The core of Lys$2 \mathrm{Arg}$ dendrimer consists of alanine amino acid residue. The inner segments contain $\varepsilon$ - or $\alpha$-part of Lys and two Arg residues. The side segments consist of $\varepsilon$-parts of Arg residues containing guanidine groups. The terminal segments contain Lys residues. Lys-2Arg dendrimer has asymmetric branching.

All NMR measurements were performed on a Bruker Avance III $500 \mathrm{MHz}$ NMR (500 MHz and $126 \mathrm{MHz}$ are frequencies for ${ }^{1} \mathrm{H}$

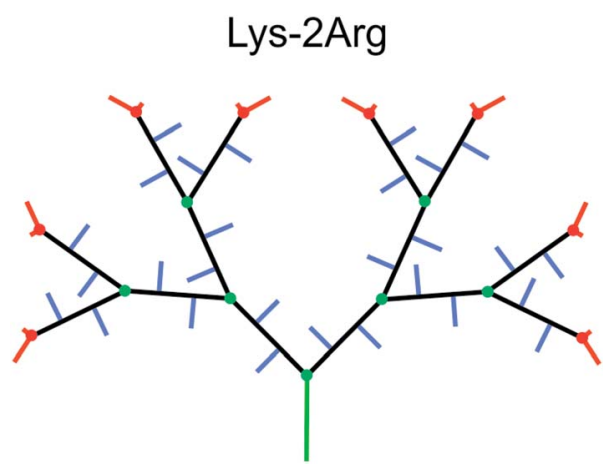

Fig. 1 Schematic structure of the second generation Lys-2Arg dendrimer. Green color marks the core, black corresponds to the main chain, violet marks the side segments, and red corresponds to the terminal segments. Green and red circles show the Lys branching points in inner and terminal segments, respectively. and ${ }^{13} \mathrm{C}$, respectively) spectrometer equipped with a standard $5 \mathrm{~mm}$ BBFO direct observation probe and a Great $1 / 60 \mathrm{~A}$ gradient system with a MIC S2 Diff/30 diffusive probe with a ${ }^{1} \mathrm{H}$ convolution compensation coil (EVT). One-dimensional ${ }^{1} \mathrm{H}$ and ${ }^{13} \mathrm{C}$ NMR spectra were recorded. We also performed twodimensional NMR experiments to confirm the Lys-2Arg dendrimer structure. ${ }^{1} \mathrm{H}^{1}{ }^{1} \mathrm{H}$ COSY, ${ }^{1} \mathrm{H}^{-13} \mathrm{C}$ HSQC, and $\mathrm{HMBC}$ spectra were obtained using standard pulse sequences. The ${ }^{1} \mathrm{H}$ spin-lattice relaxation times, $T_{1 \mathrm{H}}$, were acquired with an "inversion-recovery" pulse sequence modified by the destructive gradient pulses at the beginning of the sequence ("spoilerrecovery" sequence). ${ }^{22}$ The diffusion coefficient was measured by a stimulated echo pulse sequence with bipolar gradients to compensate for the effect of convection. ${ }^{23}$ We explored the signals from the $\mathrm{CH}_{2}$ groups chemically connected with $\mathrm{N}$ atoms to study the orientational mobility in the dendrimer by NMR relaxation.

According to Fig. 2, Lys-2Arg dendrimer has the $\mathrm{CH}_{2}-(\mathrm{N})$ groups inside the main chain, but only in those segments that contain the $\varepsilon$-part of lysine, and we consider them as "inner" groups in this study. Also, Lys-2Arg-dendrimer has the "side" $\mathrm{CH}_{2}-(\mathrm{N})$ groups of Arg residues in side segments. Methylene groups connected with protonated $\mathrm{NH}^{3+}$ groups in the terminal segments are considered "terminal" ones.

Lys-2Arg dendrimer was dissolved in $0.154 \mathrm{M} \mathrm{NaCl} \mathrm{D}_{2} \mathrm{O}$ (saline solution) at a concentration of about $1.50 \mathrm{~g} \mathrm{dl}^{-1}$.

\section{Results}

\section{${ }^{1} \mathrm{H}$ and ${ }^{13} \mathrm{C}$ spectral studies}

There are three regions in the ${ }^{1} \mathrm{H}$ NMR spectrum (Fig. 3), where peaks are observed: $4.39-3.89 \mathrm{ppm} ; 3.26-2.92 \mathrm{ppm}$ and $2.03-$ $1.22 \mathrm{ppm}$. In the first region (4.39-3.89 ppm), peaks 1-3 refer to $\mathrm{CH}$ groups in the dendrimer. The sum of the integrals for peaks 1-3 corresponds to the total content of protons in $\mathrm{CH}$ groups and has been taken as a reference. The other two regions belong to $\mathrm{CH}_{2}$ groups in different chemical environments. These are groups bonded to nitrogen-containing groups (peaks 4-6), and groups located in aliphatic parts (peaks 7-9). The integral intensity of peaks in these areas corresponds well to the chemical structure of the dendrimer (see Table 1).

According to the chemical structure (Fig. 2), the dendrimer under consideration has several types of $\mathrm{CH}$ groups located: (1) at the Lys branching points between the inner segments; (2) at the side segments; (3) at the Lys branching points between the inner and the terminal segments. However, as will be shown below, there is no exact correspondence between peaks 1-3 and types of $\mathrm{CH}$ groups.

The electronegativity of a nitrogen atom influences protons in $\mathrm{CH}_{2}$ groups and leads to their increased chemical shift with respect to other $\mathrm{CH}_{2}$ groups. This means that peaks 4-6 in the range of 3.26-2.92 ppm belong to the $\mathrm{CH}_{2}$ groups attached to nitrogen $\left(\mathrm{CH}_{2}-(\mathrm{N})\right)$. Three distinct peaks indicate the presence of $\mathrm{CH}_{2}-(\mathrm{N})$ groups in three different chemical environments. According to the chemical structure, there are $\mathrm{CH}_{2}-(\mathrm{N})$ groups in the main chain and in the side segments, and $\mathrm{CH}_{2}-(\mathrm{N})$ groups bonded to the protonated $\mathrm{NH}_{3}{ }^{+}$groups in the terminal 


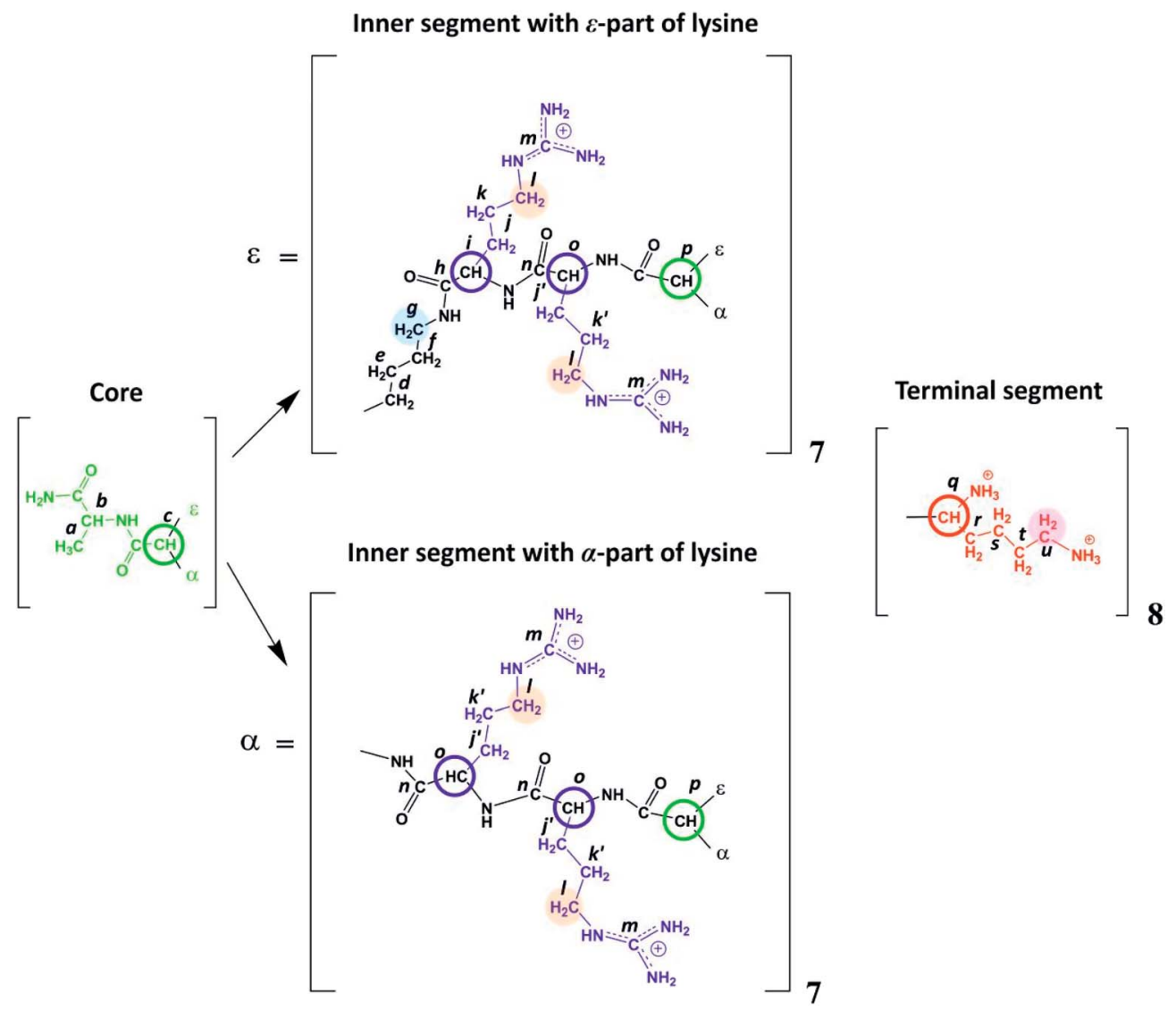

Fig. 2 Chemical structure of Lys-2Arg dendrimer. The core of Lys-2Arg dendrimer consists of alanine amino acid residue. The inner segments contain $\varepsilon$ - or $\alpha$-part of Lys and two Arg residues. The side segments consist of $\varepsilon$-parts of Arg residues containing guanidine groups. The terminal segments contain Lys residues. Green color marks the core, black corresponds to the main chain, violet indicates the side segments, and red corresponds to the terminal segments. Green and red open circles show the Lys branching points in inner and terminal segments, respectively. Blue open circles show the $\mathrm{CH}$ groups of Arg. The $\mathrm{CH}_{2}$ groups of inner segment (i.e. of $\varepsilon$-part of branched Lys) connected to $\mathrm{NH}$ groups are highlighted by light blue, $\mathrm{CH}_{2}$ groups connected to $\mathrm{NH}$ groups in side segments are highlighted by orange, $\mathrm{CH}_{2}$ groups connected to protonated $\mathrm{NH}_{3}{ }^{+}$groups in terminal segments (i.e. in $\varepsilon$-parts of terminal Lys) are highlighted by magenta.

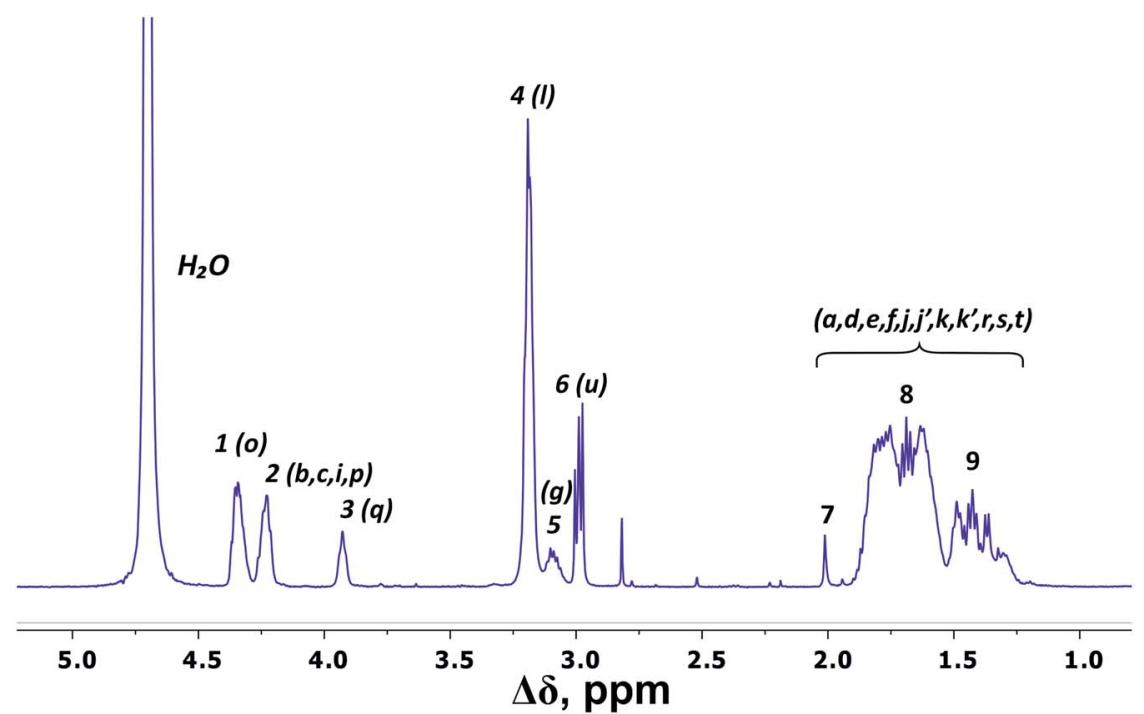

Fig. $3{ }^{1} \mathrm{H}$ NMR spectrum of Lys-2Arg dendrimer at $298 \mathrm{~K}$. The letter symbols correspond to the designations of the groups in Fig. 2 . The attribution of peaks 1-9 is shown in Table 1. 
Table 1 Chemical shift assignments and integral values

\begin{tabular}{|c|c|c|c|c|c|c|}
\hline \multirow{2}{*}{$\frac{\text { Peak number }}{1}$} & \multirow{2}{*}{$\begin{array}{l}\text { Type of group } \\
\mathrm{CH}-(\mathrm{N})\end{array}$} & \multirow{2}{*}{$\begin{array}{l}\text { Chemical shift, ppm } \\
4.34\end{array}$} & \multicolumn{2}{|c|}{ Integral value } & \multirow{2}{*}{$\begin{array}{l}\begin{array}{l}\text { Number of } \\
\text { groups }\end{array} \\
44\end{array}$} & \multirow{2}{*}{$\begin{array}{l}\text { Number of protons } \\
\text { in groups } \\
44\end{array}$} \\
\hline & & & 20.5 & 44.00 & & \\
\hline 3 & $\mathrm{CH}-(\mathrm{N})$ & 3.93 & 7.2 & & & \\
\hline 4 & $\mathrm{CH}_{2}-(\mathrm{N})$ (side $)^{a}$ & 3.19 & 60.9 & 68.9 & 28 & 56 \\
\hline 5 & $\mathrm{CH}_{2}-(\mathrm{N})$ (inner) & $3.10,3.19$ & 8.0 & & 7 & 14 \\
\hline
\end{tabular}

segments (Fig. 2). The integral value of peak 6 is proportional to the number of protons in $\mathrm{CH}_{2}-(\mathrm{N})$ groups in the terminal segments. Thus, peaks 4 and 5 refer to the side and the inner groups, respectively. Notice that the integral value of peak 5 is lower than the content of protons. Furthermore, the sum of the protons of peaks 4 and 5 is equal to 70 and the integral intensity is 68.9 (Table 1). We assume that peak 4 corresponds mainly to the signal from the side groups, and peak 5 corresponds to the signal from part of the protons of the inner groups. Then, using the ${ }^{1} \mathrm{H}^{13}{ }^{13} \mathrm{C}$ HQSC, HMBC, and ${ }^{1} \mathrm{H}^{-1} \mathrm{H}$ COSY experiments, we have shown that our assumption is true. For accuracy and consistency in using of terminology, we will associate peak 4 with the side groups (symbol $l$ in Fig. 2 and 3), and peak 5 with the inner groups (symbol $g$ in Fig. 2 and 3).

Peaks 7-9 are assigned to all other $\mathrm{CH}_{2}$ groups of Lys-2Arg dendrimer. Unfortunately, we were not able to carry out a detailed analysis in this region of the spectrum due to the overlapping of various peaks.

Fig. 4 shows the ${ }^{13} \mathrm{C}$ NMR spectrum of Lys-2Arg dendrimer. According to the chemical structure, there are five types of ${ }^{13} \mathrm{C}$ nuclei in the dendrimer: methyl $\left(\mathrm{CH}_{3}\right)$, methylene $\left(\mathrm{CH}_{2}\right)$, methine $(\mathrm{CH})$, carbonyl $(\mathrm{CO})$ and quaternary $(\mathrm{C})$. The signals in the range of 173.5-172.9 ppm belong to carbon atoms located in carboxyl groups. The quaternary carbon of the guanidine group of arginine has a chemical shift equal to $156.71 \mathrm{ppm}$. The group of peaks in the range of $54-52.95 \mathrm{ppm}$ refers to the carbon atoms in the $\mathrm{CH}$ groups. The next group of peaks in the range of 40.67-39 ppm refers to carbons of $\mathrm{CH}_{2}$ groups bonded to nitrogen atom. In the region of $31.25-21.20 \mathrm{ppm}$, there are peaks from carbons in the $\mathrm{CH}_{2}$ groups of the aliphatic part of the dendrimer.

The primary analysis of the spectrum does not allow establishing the correspondence between peaks and carbon atoms of a particular segment. For accurate correlation of peaks in the ${ }^{1} \mathrm{H}$ and ${ }^{13} \mathrm{C}$ NMR spectra, we measured two-dimensional spectra. This allowed the possibility to determine a connection between the peaks in the spectrum of (i) hydrogen atoms in neighboring $\mathrm{CH}_{n}$ groups $\left({ }^{1} \mathrm{H}^{-1} \mathrm{H} \mathrm{COSY}\right)$, (ii) carbons and hydrogens in one group $\left({ }^{1} \mathrm{H}^{13} \mathrm{C}\right.$ HSQC), and (iii) carbons and protons in adjacent groups and groups separated by two to four chemical bonds $\left({ }^{1} \mathrm{H}^{-13} \mathrm{C}\right.$ HMBC).

The detailed analysis of two-dimensional spectra is provided in ESI. $\dagger$ Here, we present only the main results and conclusions. Cross peaks $(3.19 ; 39.19)$ and $(3.09 ; 39.19)$ on the ${ }^{1} \mathrm{H}^{13}{ }^{13} \mathrm{C}$ HQSC spectrum of the dendrimer (Fig. 5) confirm that protons of the inner groups contribute to peaks 4 and 5 in Fig. 3 and Table 1. According to the ${ }^{1} \mathrm{H}^{-}{ }^{1} \mathrm{H}$ COSY spectrum (Fig. 6), protons with chemical shifts of 3.09 and $3.19 \mathrm{ppm}$ are in the same group. These two facts indicate that hydrogen nuclei in the same $\mathrm{CH}_{2}$ group have different chemical shifts (i.e., nonequivalent protons). In this case, the contribution of inner groups should be divided into two equal parts between peaks 4 and 5 .

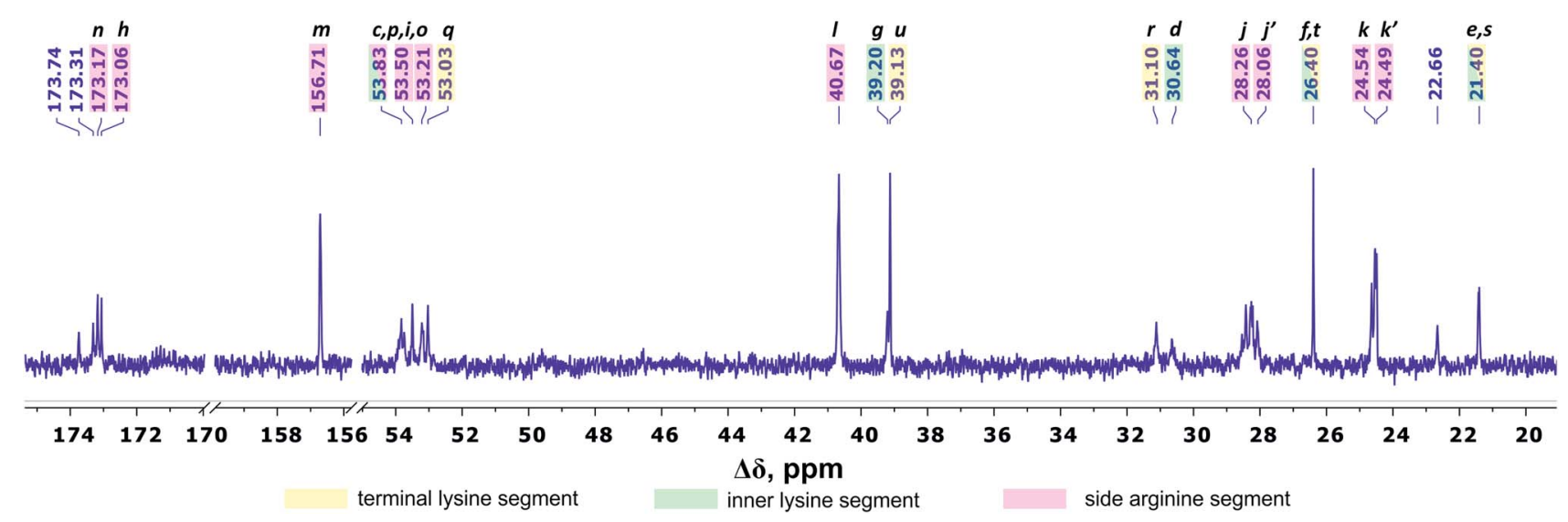

Fig. $4{ }^{13} \mathrm{C}$ NMR spectrum of Lys-2Arg dendrimer at $298 \mathrm{~K}$. The letter symbols correspond to the designations of the groups in Fig. 2. 


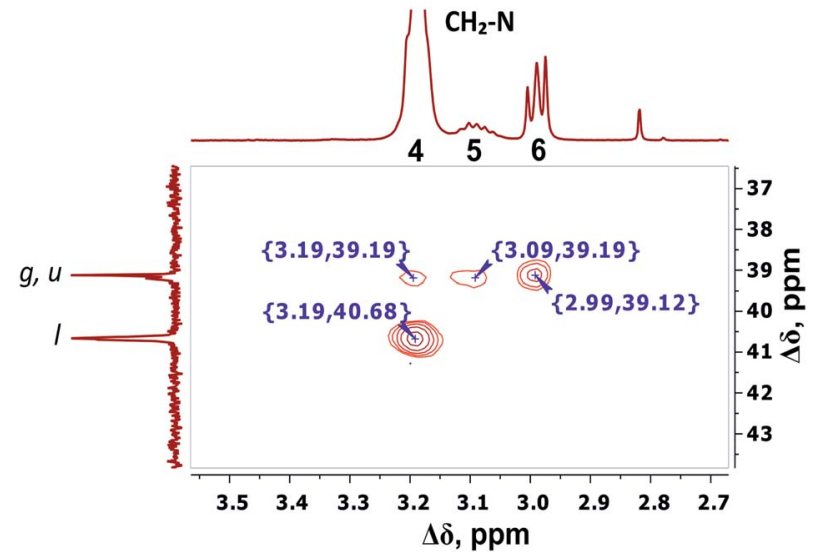

Fig. 5 Two-dimensional ${ }^{1} \mathrm{H}-{ }^{13} \mathrm{C}$ HSQC spectrum of Lys-2Arg dendrimer in the range of $3.5-2.7 \mathrm{ppm}$ at $298 \mathrm{~K}$.

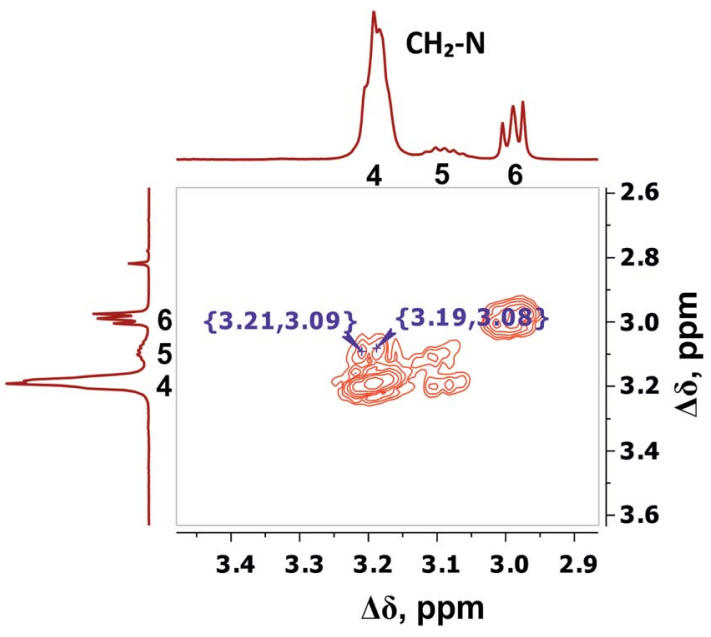

Fig. 6 Two-dimensional ${ }^{1} \mathrm{H}-{ }^{1} \mathrm{H}$ COSY spectrum of Lys-2Arg dendrimer in the range of $3.4-2.9 \mathrm{ppm}$ at $298 \mathrm{~K}$.

According to Table 1, the calculated integral value of peak 5 is actually lower than the content of protons in the inner groups. Consequently, the part of the protons in the inner groups gives a signal at peak 4 . The nonequivalence of these protons can be explained by their vicinity to the chiral carbon atom in the $\mathrm{CH}$ group of Arg (symbol $i$ in Fig. 2). Despite chirality being present in most amino acids, we did not observe such an effect in the PLL dendrimers (with Lys-2Lys and Lys repeating units) that we studied earlier. ${ }^{2,25}$ It seems that features of the chemical structure of arginine contribute to the appearance of another peak, namely peak 5 (Fig. 3), in the range of the signal from $\mathrm{CH}_{2}-(\mathrm{N})$ groups.

We conclude that 28 protons of $\mathrm{CH}$ groups of the side segments are distributed between peaks 1 and 2 as follows: seven protons (symbol $i$ in Fig. 2) give a signal at peak 2, and all other protons (symbol $o$ in Fig. 2) contribute to peak 1 . According to the cross peak $(4.25 ; 30.67)$ (Fig. 7), the protons of $\mathrm{CH}$ groups at Lys branching points between the inner segments (Fig. 2, green open circles) also contribute to the integral of peak 2 .
By analogy with $\mathrm{CH}_{2}-(\mathrm{N})$ groups, we consider that the protons at peak 3 with the smallest chemical shift of $3.94 \mathrm{ppm}$ belong to $\mathrm{CH}$ groups located at Lys branching points between the inner and the terminal segments (Fig. 2, red open circles).

According to ${ }^{1} \mathrm{H}-{ }^{13} \mathrm{C}$ HMBC data, the assignment of chemical shift at $156.71 \mathrm{ppm}$ (symbol $m$ in Fig. 4) to the carbon signal of the guanidine group is confirmed by the presence of crosspeak $(3.19 ; 156.71)$ (Fig. 7) with protons in the side $\mathrm{CH}_{2}-(\mathrm{N})$ groups in Arg residues. Also, the chemical shift (156.71 ppm) of carbon in the guanidine group is close to the chemical shift of carbon in the guanidine group in L-arginine (159.50 ppm).

Thus, the detailed analysis of the one-dimensional and twodimensional NMR spectra on ${ }^{1} \mathrm{H}$ and ${ }^{13} \mathrm{C}$ nuclei make it possible to confirm the structure of Lys-2Arg dendrimer. Moreover, the ratio of $\mathrm{CH}, \mathrm{CH}_{2}-(\mathrm{N})$ and $\mathrm{CH}_{2}$ groups in the ${ }^{1} \mathrm{H}$ NMR spectrum (Fig. 3) differs by less than $2 \%$ from the theoretically calculated one according to the structural formula (Fig. 2). Also, it is important to repeat that the inner groups possess chemically nonequivalent protons.

\section{Diffusion}

We estimated the hydrodynamic parameters of Lys-2Arg dendrimer. The diffusion coefficient is $D=1.02 \times 10^{10} \mathrm{~m}^{2} \mathrm{~s}^{-1}$. The calculation details are presented in ESI. $\dagger$ This value allows us to determine the hydrodynamic radius by using the Stokes-Einstein eqn (1):

$$
D=\frac{k_{\mathrm{B}} T}{6 \pi \eta R_{\mathrm{h}}}
$$

where $\eta$ is the solvent viscosity and $R_{\mathrm{h}}$ is the hydrodynamic radius; $T$ and $k_{\mathrm{B}}$ are temperature and Boltzmann constant, respectively.

The calculated hydrodynamic radius from eqn (1) is $1.93 \mathrm{~nm}$. It was found that the diffusion coefficient and hydrodynamic radius are the same as in Lys-2Lys dendrimer, which we obtained in our previous paper. ${ }^{24}$ In comparison with Lys-2Lys dendrimer, Lys-2Arg dendrimer has a slightly higher density equal to $0.35 \mathrm{~g} \mathrm{~cm}^{-3}$ (in contrast to $0.31 \mathrm{~g} \mathrm{~cm}^{-3}$ ). Therefore, we can conclude that the guanidine groups in side segments of Lys2Arg dendrimer do not lead to significant changes in size and density of Lys-2Arg dendrimer in comparison with Lys-2Lys dendrimer.

\section{${ }^{1} \mathrm{H}$ NMR relaxation}

Local orientational mobility in macromolecules can be studied by NMR relaxation measurements. For these studies, we considered the temperature dependence of spin-lattice relaxation for the peptide dendrimers. The spin-lattice relaxation rate for the dipole-dipole relaxation mechanism of ${ }^{1} \mathrm{H}$ nuclei is written as ${ }^{24,26-30}$

$$
\frac{1}{T_{1 \mathrm{H}}}=A_{0}\left(J\left(\omega_{\mathrm{H}}, \tau_{i}\right)+4 J\left(2 \omega_{\mathrm{H}}, \tau_{i}\right)\right),
$$

where $\omega_{\mathrm{H}}$ is the cyclic resonance frequency for ${ }^{1} \mathrm{H}$ nuclei; $A_{0}$ is a constant that does not depend on temperature and frequency; and $J$ is the spectral density that corresponds to Fourier 


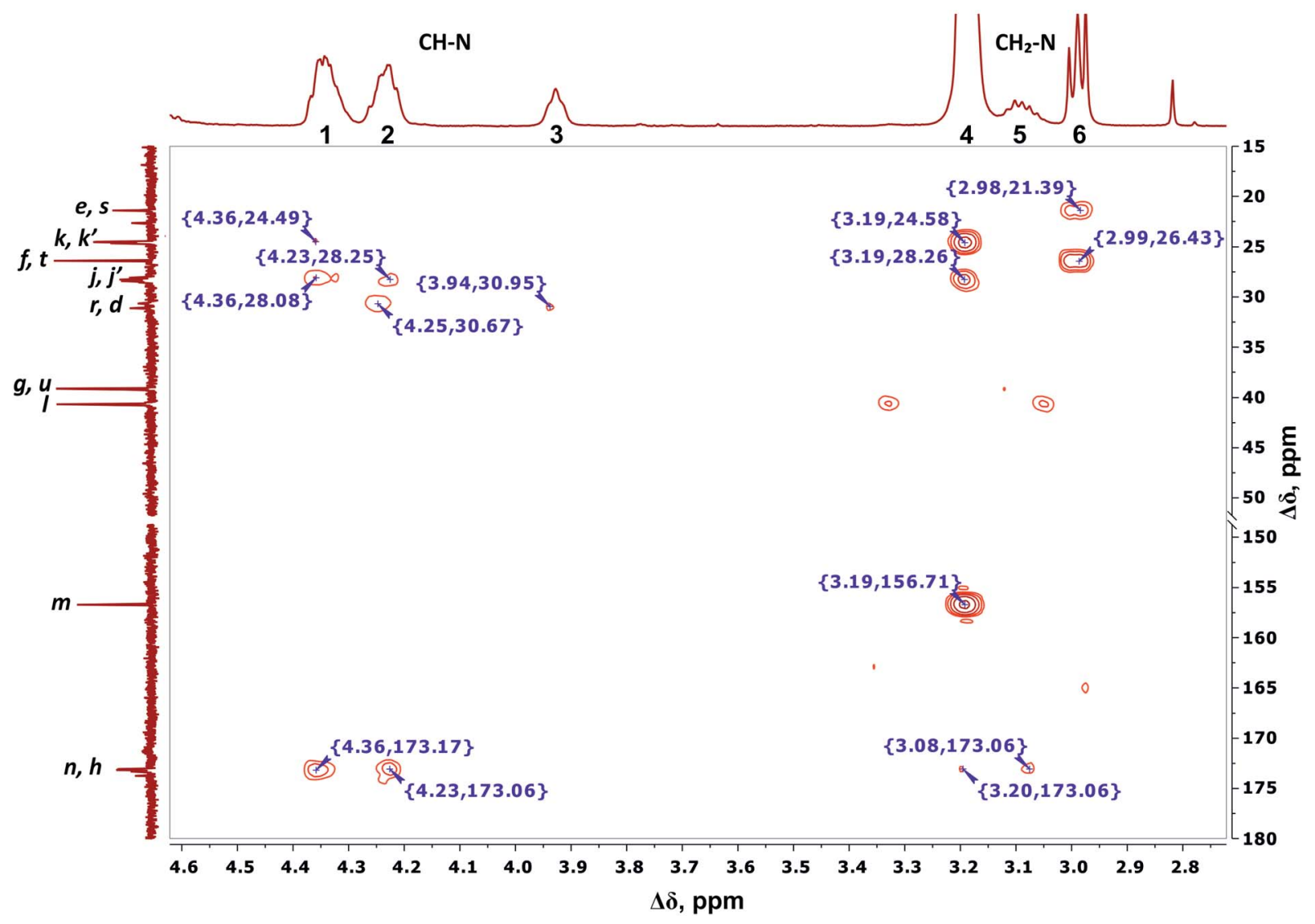

Fig. 7 Two-dimensional ${ }^{1} \mathrm{H}-{ }^{13} \mathrm{C} \mathrm{HMBC}$ spectrum of Lys-2Arg dendrimer in the range of $4.6-2.7 \mathrm{ppm}$ at $298 \mathrm{~K}$. The letter symbols correspond to the designations of the groups in Fig. 2.

transform of $P_{2}$ orientational autocorrelation functions averaged over groups contributing to a corresponding peak. In the general case, the spectral density function for ${ }^{1} \mathrm{H}$ nuclei has the form

$$
J\left(n \omega_{\mathrm{H}}, \tau_{i}\right)=\sum_{i} \frac{C_{i} \tau_{i}}{1+\left(\tau_{i} n \omega_{\mathrm{H}}\right)^{2}},
$$

where $\tau_{i}$ and $C_{i}$ are, respectively, $i$ th correlation times and their contribution to $J$, and $n=1,2$. The correlation time is determined by Arrhenius dependence

$$
\tau=\tau_{0} \exp \left(\frac{E_{\mathrm{a}}}{k_{\mathrm{B}} T}\right)
$$

where $E_{\mathrm{a}}$ is the activation energy for mobility of the chosen group.

It is important to note that spectral density for different groups of a dendrimer macromolecule cannot be described in the framework of usual simplifications that take into account one or two correlation times. The developed theory of orientational mobility in dendrimers and hyperbranched macromolecules $^{25,31-42}$ shows that local reorientation of a group in the dendrimer is determined by three main processes:

(i) Local reorientation of a given group which can be characterized by one relaxation time that is independent of the macromolecule size and the position of the group in the dendrimer; (ii) Pulsation of branches/subbranches characterized by a set of relaxation times corresponding to the rotation as a whole of the pulsating branches/subbranches with various sizes;

(iii) The rotation of the dendrimer as a whole depends only on the size of the dendrimer.

We presented a detailed description of this theory in our recent review. ${ }^{43}$ It is important to note that the developed theory is in agreement with the experimental data, in particular for

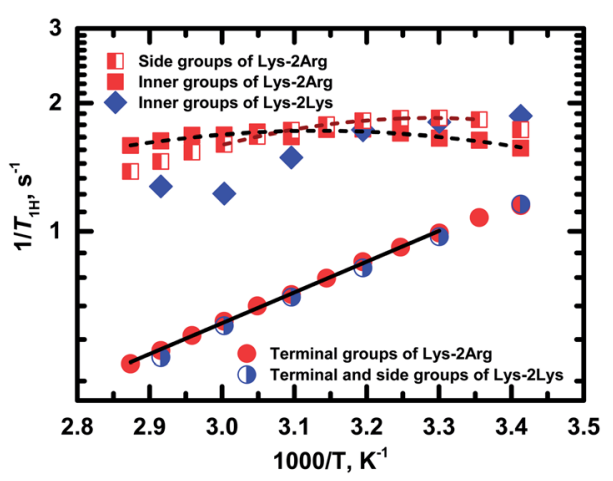

Fig. 8 Temperature dependences of the spin-lattice relaxation rate, $1 /$ $T_{1 \mathrm{H}}$, for different groups in Lys-2Arg and Lys-2Lys dendrimers. Experimental data are shown as symbols. Dash and solid lines indicate the fitting of experimental data for Lys-2Arg dendrimer by eqn (2)-(4) and Arrhenius equation, respectively. 
Table 2 Calculation of mobility parameters of $\mathrm{CH}_{2}-(\mathrm{N})$ groups in eqn (2)-(4)

\begin{tabular}{llll}
\hline $\begin{array}{l}\text { Type of } \mathrm{CH}_{2}-(\mathrm{N}) \\
\text { groups }\end{array}$ & $A_{0} \times 10^{-10}( \pm 20 \%)$ & $\tau_{0}[\mathrm{ps}]( \pm 20 \%)$ & $E_{\mathrm{a}}\left[\mathrm{kJ} \mathrm{mol}{ }^{-1}\right]( \pm 5 \%)$ \\
\hline Side $^{a}$ & 0.41 & 0.21 & 17 \\
Inner & 0.38 & 1.03 & 14 \\
Terminal & $0.38^{b}$ & $0.21^{b}$ & 14
\end{tabular}

${ }^{a} 92 \%$ side groups. ${ }^{b}$ The absence of a maximum of dependence for the terminal groups does not allow extracting $\tau_{\mathrm{c}}$ from this dependence. Thus, we used parameter $A_{0}$ for the inner groups and calculated $\tau_{0}$ by $\tau_{0}=\frac{e^{a}}{5 A_{0}}$, where $a$ is a parameter of Arrhenius fitting. This equation can be obtained from eqn (2)-(4) when $\omega \tau_{\mathrm{c}} \ll 1$. In this table the values of $\tau_{\mathrm{c}}$ are shown.

poly(acryl ether) dendrimers ${ }^{\mathbf{4 4 , 4 5}}$ where it is possible to measure $1 / T_{1 \mathrm{H}}$ for different dendrimer shells.

Fig. 8 shows the temperature dependences of $1 / T_{1 \mathrm{H}}$ for the side (peak 4), inner (peak 5) and terminal groups (peak 6).

Exponential growth of the $1 / T_{1 \mathrm{H}}$ dependence with decreasing temperature is observed for the terminal groups. The results of fitting by Arrhenius equation are shown in Table 2. Activation energy, $E_{\mathrm{a}}$, of the terminal groups equals to $14 \mathrm{~kJ} \mathrm{~mol}^{-1}$ and is similar to the $E_{\mathrm{a}}$ value for the terminal groups in Lys dendrimer. ${ }^{38}$

In the case of the side and inner groups, the maxima of $1 / T_{1 \mathrm{H}}$ dependencies have appeared. The maximum for the inner groups is at a higher temperature $(323 \mathrm{~K})$ than for the side groups ( $303 \mathrm{~K}$ ). To estimate the difference between correlation times of the side and inner groups, we approximated $1 / T_{1 \mathrm{H}}$ dependences by eqn (2)-(4) using the simplest approach with one correlation time, $\tau_{\mathrm{c}}$, which is an average relaxation time in the region of the $1 / T_{1 \mathrm{H}}$ maximum. Results of these fittings are shown in Table 2. The activation energy of the side groups is higher than $E_{\mathrm{a}}$ of inner groups but $\tau_{\mathrm{c}}$ is shorter for the side groups. For illustration, we present the calculated values of $\tau_{\mathrm{c}}$ at room temperature (Table 2). Apparently, all three processes contribute to the mobility of the inner groups; as a result, we see a wider maximum than for the side groups (Fig. 8). In the case of the side groups, the maximum should be determined only by the second process - branch pulsations. Also, an important result is that the correlation time for the terminal groups is significantly faster than $\tau_{\mathrm{c}}$ for the side and inner groups. This result is caused by the fact that the correlation time depends only on the mobility of the terminal segment, i.e., the smallest possible dendrimer branch. ${ }^{43}$ The explanation of this behavior is presented below in Discussion.

\section{Discussion}

As mentioned above, the size of Lys-2Arg dendrimer does not change in comparison with Lys-2Lys dendrimer. However, NMR relaxation of the inner and the side groups in Lys-2Arg dendrimer changed dramatically. To illustrate these changes, we compare $1 / T_{1 \mathrm{H}}$ dependences for Lys-2Arg and Lys-2Lys dendrimers in Fig. 8. The temperature dependences of $1 / T_{1 \mathrm{H}}$ for the terminal groups of both dendrimers are practically identical and possess exponential growth with a decrease of temperature. This behavior is due to the high orientational mobility of the terminal groups in both dendrimers. The opposite is true for the side groups. In Lys-2Lys dendrimers, the side groups have the same high mobility as the terminal groups. In Lys-2Arg, the temperature dependence of $1 / T_{1 \mathrm{H}}$ for the side groups has the maximum at $303 \mathrm{~K}$. The correlation time, $\tau_{\mathrm{c}}$, of the side groups is longer by several times than $\tau_{\mathrm{c}}$ of the terminal groups. This demonstrates the low mobility of the side groups. It is important to note that the shift in the position of $1 / T_{1 \mathrm{H}}$ maximum to high temperatures means the slowing down of the orientational mobility. The inner groups in Lys-2Arg dendrimer have lower mobility than the side groups because the maximum is achieved at $323 \mathrm{~K}$ and $\tau_{\mathrm{c}}$ is longer than $\tau_{\mathrm{c}}$ of the side groups.

According to the theory, the mobility of the side segments should be similar to the mobility of the terminal segments. ${ }^{24,40}$ This statement was confirmed by our recent experimental work using Lys-2Lys dendrimers. ${ }^{24}$ The opposing situation is in Lys2Arg dendrimer, where the mobility of the side groups is similar to the mobility of the inner ones. This effect is probably connected with some specific feature of guanidine groups in the side segments of Lys-2Arg dendrimer.

The obvious explanation for this effect is that there are extra interactions between the guanidine groups, with each other or with other dendrimer parts. Unfortunately, we cannot establish the nature of these guanidine group interactions by using NMR methods. However, the interaction between guanidine groups in neighboring side segments occurs due to a like-charge arginine-arginine pairing effect. ${ }^{46-49}$ This effect is absent in aqueous poly-lysine containing ammonium-terminated side chains. ${ }^{46}$ Most likely, these interactions of guanidine groups of Arg residues hinder the orientational mobility of the side segments. This effect can cause a $1 / T_{1 \mathrm{H}}$ maximum for the mobility of the side groups. Moreover, any additional interactions between dendrimer branches slow down the reorientation of these branches. As shown earlier (see review ${ }^{43}$ ), the mobility of a branch - the pulsation of a branch - as a whole makes a significant contribution to the NMR relaxation of inner groups. Thus, the same guanidine interactions can lead to a shift of $1 / T_{1 \mathrm{H}}$ maximum to higher temperatures. It is important to note that NMR relaxation has been utilized for studies of structural properties of dendrimers; for example, see our works in ref. 50-52.

Additionally, we would like to draw attention to the nonequivalent protons in the inner groups, which were found in the analysis of the NMR spectra above. On the one hand, this 
nonequivalence can be explained by the chirality of lysine and arginine amino acids. On the other hand, it is more likely caused by a slowdown in the mobility of the side segments. Thus, the conformation in which hydrogen nuclei within the same $\mathrm{CH}_{2}$ group have different chemical shifts is the predominant conformation for Lys-2Arg dendrimer. This assumption is supported by the fact that there is no similar effect in Lys-2Lys dendrimer, in which the guanidine groups are "replaced" with $\mathrm{NH}_{2}$. However, we should highlight the difference between NMR spectroscopy and relaxation data. In NMR relaxation, the correlation time values for dendrimer groups do not exceed 1 ns. However, to detect the nonequivalence of the protons of the $\mathrm{CH}_{2}$ group in an NMR spectral experiment, the lifetime of this conformation on the order of milliseconds is required.

We plan further study of the influence of the guanidineguanidine pairing effect on NMR relaxation in dendrimers by molecular dynamic simulations methods. We believe that the possibility of detecting this pairing effect in dendrimers using a relatively simple NMR relaxation method is of interest to biological applications.

\section{Conclusions}

In this work, we have synthesized and studied physicochemical properties of a new structure of second-generation poly---lysine dendrimer, in which segments (repeating units) between lysine branching points of the dendrimer consist of two linear Arg residues. This modification could be favorable for an increase of transfection by lysine based dendrimers that is important for biomedical applications. ${ }^{20}$

Lys-2Arg dendrimer was synthesized by standard solid phase peptide synthesis. The structure of the dendrimer (see Fig. 1 and 2) was established by one and two dimensional ${ }^{1} \mathrm{H}$ and ${ }^{13} \mathrm{C}$ NMR spectroscopy, including ${ }^{1} \mathrm{H}^{-1} \mathrm{H}$ COSY, ${ }^{1} \mathrm{H}_{-}{ }^{13} \mathrm{C} \mathrm{HSQC}$, and HMBC techniques as well as relaxation $\left(T_{1 \mathrm{H}}\right)$ and diffusion measurements.

NMR spectroscopy studies allowed us not only to confirm the claimed structure but also to find the presence of nonequivalence of protons in the inner $\mathrm{CH}_{2}-(\mathrm{N})$ groups, which is absent in similar Lys-2Lys dendrimer. Moreover, this circumstance should be taken into account in further study of peptide dendrimers. It will allow more accurate analysis of the ${ }^{1} \mathrm{H}$ NMR spectra and avoid misinterpretation of the integral values. $T_{1 \mathrm{H}}$ relaxation measurements showed a non-typical sharp decrease in mobility of methylene groups in side segments. For typical dendrimer topology, the mobility of the side $\mathrm{CH}_{2}-(\mathrm{N})$ groups should be close to fast mobility of the terminal $\mathrm{CH}_{2}-(\mathrm{N})$ groups; for instance, see the results for Lys-2Lys dendrimer in ref. 24 In the case of Lys-2Arg dendrimer, the mobility of side segments is similar to the mobility of inner segments. Our estimation of the correlation times, $\tau_{\mathrm{c}}$, at room temperature for $\mathrm{CH}_{2}-(\mathrm{N})$ groups in the inner, side, and terminal segments gave the following values: $290 \mathrm{ps}, 200 \mathrm{ps}$, and $60 \mathrm{ps}$, respectively. This unexpected $T_{1 \mathrm{H}}$ behavior is most likely caused by well-known guanidineguanidine pairing effect in water, which leads to entanglements between dendrimer branches. Thus, the unique structure of the dendrimer creates the possibility to identify the presence of entanglements inside the macromolecule using fairly simple measurements of NMR relaxation. We believe that this finding will be important not only to establish the structure of dendrimers but also to study a wider class of hyperbranched macromolecules.

\section{Conflicts of interest}

There are no conflicts to declare.

\section{Acknowledgements}

The NMR measurements were carried out in the Center for Magnetic Resonance of Research Park of St. Petersburg State University. This work is supported by the Russian Science Foundation (grants No. 19-13-00087). E. L. and N. N. S. are thankful for the Researcher Mobility Grant of the Academy of Finland (No. 318608) and the travel grant provided by Saint Petersburg State University (Event 6, ID 36154550). I. M. N. was supported by grant 08-08 of the Government of Russian Federation.

\section{References}

1 A. Samad, I. Alam and K. Saxena, Curr. Pharm. Des., 2009, 15, 2958-2969.

2 E. Abbasi, S. F. Aval, A. Akbarzadeh, M. Milani, H. T. Nasrabadi, S. W. Joo, Y. Hanifehpour, K. NejatiKoshki and R. Pashaei-Asl, Nanoscale Res. Lett., 2014, 9, 247.

3 S. Mutalik, H. S. Parekh, Y. G. Anissimov, J. E. Grice and M. S. Roberts, Skin Pharmacol. Physiol., 2013, 26, 127-138.

4 B. Noriega-Luna, L. A. Godínez, F. J. Rodríguez, A. Rodríguez, G. Zaldívar-Lelo de Larrea, C. F. Sosa-Ferreyra, R. F. MercadoCuriel, J. Manríquez and E. Bustos, J. Nanomater., 2014, 2014, 1-19.

5 M. Ghaffari, G. Dehghan, F. Abedi-Gaballu, S. Kashanian, B. Baradaran, J. E. N. Dolatabadi and D. Losic, Eur. J. Pharm. Sci., 2018, 122, 311-330.

6 L. M. Bronstein and Z. B. Shifrina, Chem. Rev., 2011, 111, 5301-5344.

7 L. Palmerston Mendes, J. Pan and V. Torchilin, Molecules, 2017, 22, 1401.

8 S. S. Santos, R. V. Gonzaga, J. V. Silva, D. F. Savino, D. Prieto, J. M. Shikay, R. S. Silva, L. H. A. Paulo, E. I. Ferreira and J. Giarolla, Can. J. Chem., 2017, 95, 907-916.

9 J. Yang, Q. Zhang, H. Chang and Y. Cheng, Chem. Rev., 2015, 115, 5274-5300.

10 T. Kuanga, D. Fub, L. Changb, Z. Yangb, Z. Chenc, L. Jine, F. Chenb and X. Peng, Curr. Org. Chem., 2016, 20, 1820-1826.

11 T. Okuda, A. Sugiyama, T. Niidome and H. Aoyagi, Biomaterials, 2004, 25, 537-544.

12 A. Kwok, G. A. Eggimann, M. Heitz, J. L. Reymond, F. Hollfelder and T. Darbre, ChemBioChem, 2016, 17, 22232229.

13 C. Liu, X. Liu, P. Rocchi, F. Qu, J. L. Iovanna and L. Peng, Bioconjugate Chem., 2014, 25, 521-532. 
14 J. B. Kim, J. S. Choi, K. Nam, M. Lee, J.-S. Park and J.-K. Lee, J. Controlled Release, 2006, 114, 110-117.

15 T. Kim, C. Z. Bai, K. Nam and J. Park, J. Controlled Release, 2009, 136, 132-139.

16 H. Hirose, T. Takeuchi, H. Osakada, S. Pujals, S. Katayama, I. Nakase, S. Kobayashi, T. Haraguchi and S. Futaki, Mol. Ther., 2012, 20, 984-993.

17 K. Luo, C. Li, L. Li, W. She, G. Wang and Z. Gu, Biomaterials, 2012, 33, 4917-4927.

18 T. Kim, J. Baek, C. Zhe Bai and J.-S. Park, Biomaterials, 2007, 28, 2061-2067.

19 X. Liu, C. Liu, J. Zhou, C. Chen, F. Qu, J. J. Rossi, P. Rocchi and L. Peng, Nanoscale, 2015, 7, 3867-3875.

20 A. Kwok, G. A. Eggimann, J.-L. L. Reymond, T. Darbre and F. Hollfelder, ACS Nano, 2013, 7, 4668-4682.

21 S. Falkovich, D. Markelov, I. Neelov and A. Darinskii, J. Chem. Phys., 2013, 139, 064903.

22 G. H. Sorland, in Dynamic Pulsed-Field-Gradient NMR, Springer-Verlag Berlin, Heidelberger Platz 3, D-14197 Berlin, Germany, 2014, vol. 110, pp. 169-190.

23 A. Jerschow and N. Müller, J. Magn. Reson., Ser. A, 1996, 123, 222-225.

24 N. N. Sheveleva, D. A. Markelov, M. A. Vovk, M. E. Mikhailova, I. I. Tarasenko, I. M. Neelov and E. Lähderanta, Sci. Rep., 2018, 8, 1-7.

25 D. A. Markelov, S. G. Falkovich, I. M. Neelov, M. Y. Ilyash, V. V. Matveev, E. Lähderanta, P. Ingman and A. A. Darinskii, Phys. Chem. Chem. Phys., 2015, 17, 32143226.

26 A. Abragam, The principles of nuclear magnetism, Clarendon Press; Oxford University Press, Oxford [Oxfordshire], New York, 1983.

27 N. Bloembergen, E. M. Purcell and R. V. Pound, Phys. Rev., 1948, 73, 679-712.

28 I. Solomon, Phys. Rev., 1955, 99, 559-565.

29 V. I. Chizhik, Y. S. Chernyshev, A. V. Donets, V. V. Frolov, A. V. Komolkin and M. G. Shelyapina, Magnetic Resonance and Its Applications, Springer, Cham, 2014.

$30 \mathrm{M}$. H. Levitt, Spin dynamics: basics of NMR, Chichester, England, Hoboken, NJ, 2nd edn, 2008.

31 Y. Y. Gotlib and D. A. Markelov, Polym. Sci., Ser. A, 2004, 46, 815-832.

32 O. V Shavykin, I. M. Neelov and A. A. Darinskii, Phys. Chem. Chem. Phys., 2016, 18, 24307-24317.

33 M. Dolgushev, S. Schnell and D. A. Markelov, Appl. Magn. Reson., 2017, 48, 657-671.
34 D. A. Markelov, F. Fürstenberg and M. Dolgushev, Polymer, 2018, 144, 65-71.

35 Y. Y. Gotlib and D. A. Markelov, Polym. Sci., Ser. A, 2007, 49, 1137-1154.

36 D. A. Markelov, S. V. Lyulin, Y. Y. Gotlib, A. V. Lyulin, V. V. Matveev, E. Lahderanta and A. A. Darinskii, J. Chem. Phys., 2009, 130, 1-9.

37 I. Neelov, S. Falkovich, D. Markelov, E. Paci, A. Darinskii and H. Tenhu, in Dendrimers in Biomedical Applications, The Royal Society of Chemistry, 2013, pp. 99-114.

38 I. M. Neelov, D. A. Markelov, S. G. Falkovich, M. Y. Ilyash, B. M. Okrugin and A. A. Darinskii, Polym. Sci., Ser. C, 2013, 55, 154-161.

39 D. A. Markelov, M. Dolgushev, Y. Y. Gotlib and A. Blumen, J. Chem. Phys., 2014, 140, 244904.

40 M. Dolgushev, D. A. Markelov, F. Fürstenberg and T. Guérin, Phys. Rev. E, 2016, 94, 012502.

41 D. A. Markelov, A. N. Shishkin, V. V Matveev, A. V Penkova, E. Lähderanta and V. I. Chizhik, Macromolecules, 2016, 49, 9247-9257.

42 J. Grimm and M. Dolgushev, Phys. Chem. Chem. Phys., 2016, 18, 19050-19061.

43 D. A. Markelov, M. Dolgushev and E. Lähderanta, in Annual Reports on NMR Spectroscopy, ed. G. A. Webb, Academic Press, 2017, vol. 91, pp. 1-66.

44 L. F. Pinto, R. Riguera and E. Fernandez-Megia, J. Am. Chem. Soc., 2013, 135, 11513-11516.

45 L. F. Pinto, J. Correa, M. Martin-Pastor, R. Riguera and E. Fernandez-Megia, J. Am. Chem. Soc., 2013, 135, 1972-1977.

46 J. Vondrášek, P. E. Mason, J. Heyda, K. D. Collins and P. Jungwirth, J. Phys. Chem. B, 2009, 113, 9041-9045.

47 J. Gao, C. Tang, M. A. Elsawy, A. M. Smith, A. F. Miller and A. Saiani, Biomacromolecules, 2017, 18, 826-834.

48 D. Lee, J. Lee and C. Seok, Phys. Chem. Chem. Phys., 2013, 15, 5844-5853.

49 C. P. Schneider, D. Shukla and B. L. Trout, J. Phys. Chem. B, 2011, 115, 7447-7458.

50 D. A. Markelov, V. V. Matveev, P. Ingman, M. N. Nikolaeva, A. V. Penkova, E. Lahderanta, N. I. Boiko and V. I. Chizhik, Sci. Rep., 2016, 6, 24270.

51 V. V Matveev, D. A. Markelov, S. V Dvinskikh, A. N. Shishkin, K. V Tyutyukin, A. V Penkova, E. A. Tatarinova, G. M. Ignat'eva and S. A. Milenin, Sci. Rep., 2017, 7, 13710.

52 D. A. Markelov, V. V. Matveev, P. Ingman, M. N. Nikolaeva, E. Lähderanta, V. A. Shevelev and N. I. Boiko, J. Phys. Chem. B, 2010, 114, 4159-4165. 